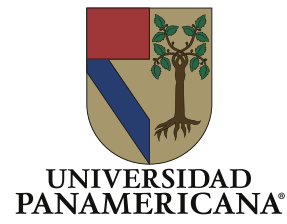

ScienceDirect

BIOETHICS UPdate 4 (2018) 87-91
BIOETHICS UPdate

www.elsevier.es/bioethicsupdate

\author{
Editorial
}

\title{
The philosophy of palliative care
}

\author{
La filosofía de los cuidados paliativos
}

\author{
Evandro Agazzi \\ Interdisciplinary Center for Bioethics, Panamerican University of Mexico, Mexico City, Mexico
}

\section{Introduction}

The adjective "palliative" has in ordinary language a rather negative flavour, expressing the idea of something that is only superficially or apparently useful to repair a certain damage but leaves it actually unaffected. This is probably the reason for its only recent circulation in medicine. The increasing interest for the "palliative care", however, is a good indication of the more serious consideration it deserves, for reasons that we could briefly outline as follows. Two principles are often presented as the most fundamental in the debates of medical ethics: One is that of the "sanctity of life" (allegedly the supreme principle of traditional medical ethics), which we can express in more "secularized" words as the principle of the "absolute respect for life". The other is that of the "quality of life" (considered as the supreme principle of modern medical ethics). Since there cannot be two "supreme" principles, it follows that one must be considered as subordinate to the other in those decisions where they come to a conflict. The different schools in bioethics often distinguish themselves for giving the supremacy to one of these two principles over the other, and this difference implicitly reflects the way of considering what is the fundamental goal of medicine. According to a certain view, this fundamental goal is the fight against death, that has pushed the progress of discovery of medicaments, treatments and surgeries capable

E-mail address: evandro.agazzi@gmail.com 
of stopping the course of certain pathologies before it comes to its "natural" end, that is, to the death of the patient. In this perspective, it is said, for example, that one "owes life to penicillin", or that "this surgery saved my life", or that "his doctor saved his life". It seems also obvious, within this perspective, that avoiding death deserves paying whatever price, in terms of money (for those who can afford it), or in terms of pains or other forms of suffering. This "heroic" view of medicine is seldom advocated today, and its intrinsic limitations have been expressed through the criticism of the "therapeutic obstinacy" that consists in submitting the patient to treatments whose effect can be only a modest extension of his survival at the price of considerable suffering.

We are not going to enter the complicated discussion regarding the possibilities of measuring the amount of suffering of the patient and to establish which would be the "right" amount of his life extension that could counterbalance it. We rather want to call attention on two points. The first is that, if we accept to qualify the fight against death as the fundamental goal of medicine, we must recognize that medicine is a frustrating enterprise that never can attain its goal, since all humans inexorably die. The most that medicine can do is "postponing" death extending not so much life, but rather survival, that is, the continuation of the purely biological life. Here comes the second point. This segment of biological life that will be conquered by applying the heroic treatment will be affected by a considerable amount of suffering and the question is whether it is reasonable, sensible, wise, to accept such a "compensation". It is obvious that such judgments regard the "quality of life" of the patient during the period of survival, and the possibility is clearly open that the answer to the question be that it is not wise to pay such a price. In this case the principle of the quality of life would be prevalent over the principle of the preservation of life.

\section{The pursuit of health}

We intentionally disregard now the variety of prices and burdens that can accompany the prolongation of survival considered here, and will limit our consideration only to "suffering" understood in strictly medical terms, in order to delimit the meaning of the quality of life to what can be encompassed within medicine. This move is not simply methodological but corresponds to a characterization of the goal of medicine that was truly "classical" and did not identify this goal with a fight against death. This classical definition (that we frequently find, for instance, in Aristotle) is that the goal of medicine is health. The concept of health has a very rich meaning and a variety of analogical applications; even in the domain of medicine it has received in 
recent times certain more or less official definitions that we are not going to consider here, but we can say that, if applied to the condition of a single individual, it could be considered equivalent with the concept of a good quality of life. If we assume the principle of securing the best quality of life as the fundamental principle of medicine, the principle of preserving life necessarily follows, because it is impossible to secure a good quality of life to someone who is not alive. Therefore, preserving the life of a patient is a prerequisite for securing his good quality of life. However, the goal of looking for a good quality of life still holds also in those cases in which life is not at risk and in fact the great majority of the pathologies medicine is concerned with are not deadly diseases. Moreover, the fact of abandoning the centrality of the fight against death has produced an increased attention for the suffering that normally accompanies the end of life treatments, and suffering itself has become a specialized domain of medical investigation and practice. The expansion of the medical and bioethical attention from the optics of "curing" to the optics of "caring" is perhaps the clearest confirmation of the fruitfulness of deepening the principle of the quality of life. It is precisely within this framework that the significance and importance of the palliative care can be appreciated.

\section{The palliative care}

Though the palliative care is very often applied to terminal patients, especially those affected by cancer, it helps logical clarity to stress that it is independent from the consideration of death. This point is suitably mentioned in the short definition of this care present in the Statutes of the European Association for Palliative Care, where it is stated that "this care respects life and considers death as a natural process. Its goal is not that of accelerating or differing death, but that of preserving the best possible quality of life until the end". The importance of this clarification resides in the fact that the palliative care does not interfere with the therapies that tend to differ death, but can and often should be applied parallel to them and also be continued after the moment where the "therapeutic" treatments are discontinued because they are clearly useless. The explicit mention of the quality of life is also significant, because it opens the space to a variety of actions that overstep the technical horizon of medicine. This is in keeping with the broadening of the notion of quality of life and of care that has occurred in the last decades, and which one can find, for example, in the definition of palliative care proposed by the World Health Organization or (with equivalent terms) by the National Cancer Institute of the USA (where we read "Palliative care is care given to improve the quality of life of patients who have a serious or life-threatening disease, such as cancer. Palliative care is an approach to care that addresses the 
persons as a whole, not just their disease. The goal is to prevent or treat, as early as possible, the symptoms and side effects of the disease and its treatment, in addition to any related psychological, social, and spiritual problems").

In these characterizations of the palliative care we can find a harmonization of the two fundamental task that common sense attributes to medicine, namely, the fight against death and the fight against suffering, to which an equal importance is attributed. The pursuit of both goals goes hand in hand, because the efforts for controlling and diminishing pain and suffering are active also during the period in which the therapeutic treatments aiming at curing the disease are fully practiced, but they continue also when such treatments are abandoned because they are clearly unsuccessful, and this because the patient is still alive and deserves being attended by medicine (as far as the control of his suffering is concerned) during what remains of his life span. This because of his dignity as human being that remains intact in all conditions of his existence. In addition, the patient deserves special compassion owing to his state of fragility that demands protection also independently of the fact that he is close to death. This is by no means an irrational attitude but simply corresponds to the awareness that a terminal patient is still a human being having his dignity.

It is easy to see how these reflections are far from the approach of euthanasia, which rests on two presuppositions. The first is that the only serious task of medicine is the fight against death and, therefore, that it has no sense to continue with medical treatments when this battle is lost. The second is that the feeling of compassion we perceive in the presence of a suffering terminal patient is the morally right reason for putting an end to this suffering and ending his life can easily attain this. It is really surprising that one does not realize that what we would do is not killing the suffering, but killing the patient. Applying palliative care, on the contrary, amounts to continuing with the other goal of medicine, that is, with controlling suffering, even by using means that could accelerate the imminent death, because this acceleration is only an unwanted consequence of a right action.

It is worth noting that this approach avoids the conflict between the two fundamental tasks of medicine mentioned above, none of which is considered absolute or supreme: the fight against death cannot impose acceptance of intolerable suffering, and fight against suffering cannot impose termination of life. As in many cases in which different principles or values risk to come to conflict, "optimization" appears as the wise solution: none of them will be fully satisfied, but none will be unduly sacrificed. The euthanasia solution would attain the disappearance of 
suffering by annihilating the defence of life. Even extreme measures of palliative care, such as terminal sedation or interruption of artificial life-sustaining procedures are not equivalent with a killing action.

These are not simply logical subtleties; they have to do with a fundamental condition for a good patient-doctor relation, on which so much stress is duly laid today. This condition is full confidence whose minimal requirement is that I must be sure that my doctor never will kill me. The general application of this obvious principle is that no doctor would kill his patient and this necessarily excludes euthanasia practiced by any doctor on any patient. The question of medically assisted suicide is very special and is not of our concern here, especially because it entails the previous very complex issue of the ethical legitimacy of suicide itself.

A last remark. The most recent definitions of palliative care include, besides the strictly medical requirements of a good quality of life, several other dimensions that concern the psychological, social and spiritual aspects of a person's existence. This means that from within medicine the awareness gushes of an opening towards non-medical approaches even to precise medically definable situations, and this calls into play several contributions from disciplines and persons different from medical doctors. This phenomenon is to a certain extent a novelty but actually corresponds to a good understanding and deepening of a maxim often repeated but perhaps seldom applied in practice, that is, that medicine does not care or cure a disease but a patient, a human being. Therefore, all the dimensions of the human person have more or less important relations and influence on all events of her life, including her pathologies. 